\title{
Evidence for the role of the diabatic heating in synoptic scale processes: a case study example
}

\author{
M. L. Martin, M. Y. Luna, F. Valero \\ Dpto. Astrofísica y CC. de la Atmósfera, Facultad de CC. Fisícas, Universidad Complutense de Madrid, 28040 Madrid, Spain
}

Received: 9 November 1995 / Revised: 18 November 1996 / Accepted: 28 November 1996

\begin{abstract}
The quasigeostrophic theory is used to address the role of diabatic forcing in synoptic scale processes over Iberia. A parametrization of diabatic heating is obtained in terms of a thermodynamic variable called the ice-liquid water potential temperature which is conservative under all phase changes of water. A case study objectively selected by means of a rotated principal component analysis over the diabatic field is analyzed to test the proposed parametrization. This study highlights the fact that the magnitudes of diabatic forcing and dynamic forcing are very nearly the same throughout the troposphere. The results also show that the composite diabatic heating is a better representation for both cloudiness and precipitation fields than the dynamic forcing.
\end{abstract}

\section{Introduction}

Accurate prediction of the onset, end, areal extent, and amount of precipitation has long been one of the primary goals of weather forecasting. Precipitation forecasts are based upon the knowledge that the upward displacement of saturated air parcels will produce precipitation. A number of investigators (Yip and Cho, 1982; Chang et al., 1984; Smith et al., 1984; Smith, 1987) have produced evidence that forecast precipitation amounts and updraft strengths are considerably underestimated by dynamic models that ignore the effects of condensation occuring in synoptic scale ascent regions.

Vertical motions associated with traveling baroclinic systems are able to be diagnosed from quasigeostrophic theory. From the classical quasigeostrophic omega equation, vertical motions are forced by vertical differ-

Correspondence to: F. Valero ential vorticity advection and the Laplacian of thermal advection (Holton, 1972). Trenberth (1978) pointed out that in the middle troposphere these two forcing effects often nearly cancel each other. He combined these two forcing effects mathematically and estimated vertical motions from a weather map containing thickness and vorticity contours. Hoskins et al. (1978) presented a more general formulation of the complete geostrophic forcing terms in the omega equation including the term ignored by Trenberth. They showed that the right-hand side of the omega equation could be written in a more compact way using the $\mathbf{Q}$ vector analysis, by which the vertical motion tends to be upward when the field of $\mathbf{Q}$ vectors is convergent. Divergence of $\mathbf{Q}$ vectors is proportional to the three-dimensional Laplacian or curvature of the omega field under assumptions that the flow is adiabatic, in hydrostatic balance and that advections are accomplished solely by the geostrophic winds (Chen et al., 1988). An interpretation of the $\mathbf{Q}$ vector is that it provides an approximate picture of the ageostrophic horizontal wind in the lower branch of the circulation that develops in order to maintain thermal wind balance in an evolving synoptic disturbance. By virtue of this scheme, in the lower troposphere, the $\mathbf{Q}$ vector would point toward the region of ascent while in the high troposphere, the direction of the $\mathbf{Q}$ vector would be opposite to the ageostrophic motion.

The obvious disadvantage is that the quasigeostrophic forcing does not incorporate vertical motion produced by diabatic heating or cooling. In fact, because the $\mathbf{Q}$ vector analysis is obtained in adiabatic conditions, this latent heat release is not taken into account. On the synoptic scale, diabatic heating appears to be very important because widespread ascent of saturated air and its associated latent heat release will tend to reinforce the ascent. In agreement with Hoskins and Pedder (1980), we think that the absence of diabatic processes in quasigeostrophic diagnosis might be a drawback.

The importance of diabatic effects on synoptic scale processes over Iberia is investigated in this study within 
the quasigeostrophic theory framework. A parametrization of diabatic heating is proposed, which is described in Sect. 2. To gain insight into the skills of parametrization, some meteorological products are computed for a case study objectively selected by a method described in Sect. 3. In Sect. 4, the proposed parametrization is tested on the case study developed in Sect. 3 in order to examine its ability to describe precipitation and cloudiness fields. Finally, the results of this study are discussed in Sect. 5.

\section{Parametrization of diabatic heating}

Quasigeostrophic theory has been the cornerstone of dynamical meteorology and has provided the conceptual basis for understanding the behavior of extratropical synoptic systems. Within the framework in this theory, is the well-known omega equation for estimating vertical motion. If the diabatic effects are not neglected in the thermodynamic equation, then omega equation includes a further term on the right-hand side which is proportional to the horizontal Laplacian of the diabatic heating. Therefore, the quasigeostrophic omega equation with diabatic heating included, can be rewritten according to Bosart (1985) as:

$$
\left(\sigma \nabla_{p}^{2}+f_{0}^{2} \frac{\partial^{2}}{\partial p^{2}}\right) \omega=-2 \nabla_{p} \cdot \mathbf{Q}-\frac{R}{p C_{p}} \nabla_{p}^{2} J
$$

A list of symbols is provided in Appendix A. In general, $J$ is the rate of heating per unit mass due to radiation, conduction, and latent heat release; in this study, $J$ is limited to latent heat release. Note that the forcing terms do really indicate that upward motion would be favored in regions where the field of $\mathbf{Q}$ vectors is convergent and diabatic heating is positive.

In this section an expression for the diabatic forcing, based on the entropy state function for a system of cloudy air, which retain latent heat release associated with processes involving ice phase changes is now developed.

Using the entropy form of the first law of the thermodynamics, heat flow may be related with specific entropy, $s$, by means of:

$\frac{J}{T} \equiv \frac{d s}{d t}$.

For a closed adiabatic system of dry air, the entropy change is given by

$d s=C_{p} d \ln \theta=0$.

Thus, if the system consists only of dry air, the dry potential temperature, is a constant. If water vapor is included, the potential temperature of dry air is not suitable to characterize the system. Instead, the potential temperature of moist air, $\theta_{m}$, should be used which is conserved when water vapor is included (Iribarne and Godson, 1973; Lips and Hemler, 1980). If liquid water is taken into account, the liquid water potential temperature, $\theta_{1}$, defined by Betts (1973), must be used. Nevertheless, it would be more desirable to extend the definition of $\theta_{1}$ and include the ice phase since consideration of ice is essential for deep convection. Tripoli and Cotton (1981) defined an ice-liquid water potential temperature, $\theta_{i l}$, which they considered to be conserved even under nonequilibrium conditions. They based the definition of $\theta_{i l}$ on an approximated form of the thermodynamic energy equation and made some further approximations to obtain the following expression:

$\theta_{i l}=\theta\left[1-\frac{L_{l v}\left(T_{0}\right) r_{l}}{C_{p} \max (T, 253)}-\frac{L_{i v}\left(T_{0}\right) r_{i}}{C_{p} \max (T, 253)}\right]$.

$\theta_{i l}$ is conserved under all phase changes of water if reversibility is assumed (i.e., for non-precipitating systems). This temperature is an extension of the liquid water potential temperature and reduces to $\theta$ in subsaturated conditions. Moreover, $\theta_{i l}$ varies continuously from clear air to cloudy air.

Because it is often desirable to handle a thermodynamic variable which is conservative under adiabatic liquid and ice transformations, the variable $\theta_{i l}$ was defined by Tripoli and Cotton (1981) so that its internal variations are zero. Variations in $\theta_{i l}$ occur only due to external changes of $r_{i}$ or $r_{l}$. Therefore, it is evident that precipitation will affect $\theta_{i l}$.

Hauf and Höller (1987) analyzed the relationship between entropy change and thermodynamic variables by which the entropy change can be related for a cloud system to $\theta_{i l}$ as:

$d s=C_{p} d \ln \theta_{i l}$. that:

By introducing this expression in Eq. (2), it follows

$J=T C_{p} \frac{d \ln \theta_{i l}}{d t}$.

Furthermore, by inserting Eq. (6) into Eq. (1), the omega equation can be rewritten as:

$\left(\sigma \nabla_{\mathrm{p}}^{2}+f_{0}^{2} \frac{\partial^{2}}{\partial p^{2}}\right) \omega=-2 \nabla_{p} \cdot \mathrm{Q}-\frac{R}{p} \nabla_{p}^{2}\left(T \frac{d \ln \theta_{i l}}{d t}\right)$.

We may then notice that a synergistic interaction between dynamics and thermodynamics can occur. By dynamics, we are referring to processes associated with quasigeostrophic forcing $\left(-2 \nabla_{p} \cdot \mathrm{Q}\right)$ and by thermodynamics to processes related to convective conditions.

\section{Selecting a case study}

\subsection{Data}

The data set consists of surface rainfall and upper-air measurements throughout the year 1990. The synoptic network of 90 rain gauges measuring 24-h accumulated precipitation was used to collect distributions of rainfall overall Iberia. Twice daily soundings at 0000 and 1200 UTC were taken at the seven stations shown in Fig. 1. Numerical computations for both dynamic forcing and diabatic forcing terms ( $G$ and $D$, respectively, hereafter) were performed from interpolated state vari- 


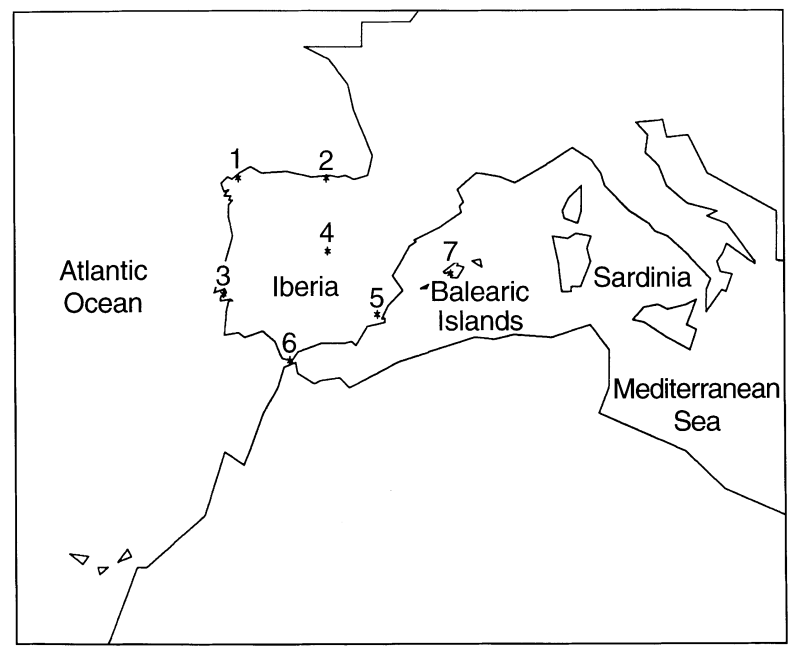

Fig. 1. Map with geographical names referred to in the text indicating location of the seven sounding stations in Iberia by numbers: $1, \mathrm{La}$ Coru ña 2, Santander; 3, Lisbon; 4, Madrid; 5, Murcia; 6, Gibraltar; 7, Palma de Mallorca

ables on a cartesian coordinate grid with a mesh size of $2^{\circ}$ latitude by $2^{\circ}$ longitude in an area encompassing the Iberian Peninsula and the Balearic Islands. The sounding data from the seven locations were objectively interpolated by moving averages (Ripley, 1981) in order to build up such a regular grid. This computationally simple, weighted-averaging method assigns a weight to a datum solely as a known function of distance between datum and grid point. Derivatives appearing in terms are approximated using centered differences of dependent variables over finite space and time intervals. Finite differences are discrete approximations to continuous derivatives $D$ and $G$ were computed for every day of the year 1990 and for a number of mandatory levels in each grid-point. Table 1 shows space-time averages of $D$ and $G$ absolute values at $850,700,500,400$ and $250 \mathrm{hPa}$. It is worth emphasizing that in general the order of magnitude is the same for the two types of forcing and that the diabatic forcing is somewhat greater than the dynamic one below the $500 \mathrm{hPa}$ level. The difference between dynamic and diabatic forcing reaches its maximum at about $700 \mathrm{hPa}$. This is the reason why we focus our analysis on that level.

\subsection{Criterion of selection}

To avoid bias, the selection of a case study should not be made subjectively. We might objectively draw a case by a random method but in this case the selected $D$ field

Table 1. Space-time averaged absolute values for dynamic $(G)$ and diabatic $(D)$ forcings $\left(\times 10^{-17} \mathrm{hPa}^{-1} \mathrm{~s}^{-3}\right)$ at several mandatory levels (hPa)

\begin{tabular}{llllll}
\hline Level & 850 & 700 & 500 & 400 & 250 \\
\hline$G$ & 2.7 & 3.6 & 7.2 & 7.5 & 7.4 \\
$D$ & 3.6 & 6.1 & 3.4 & 3.3 & 3.1 \\
\hline
\end{tabular}

might be poorly representative. That is the reason why in this work the case study has been chosen through an objective (automated) classification scheme based on principal component analysis (PCA) trying to find a daily weather situation, which will most closely resemble the first rotated principal component of the $D$ field. The case study has been chosen from the complete $D$ data set in order to validate the diabatic forcing term in Eq. (7). PCA has proven to be a reliable method for data reduction and for examining the variance structure. PCA applied to spatial data, known as S-mode decomposition (Richman, 1988), enables patterns to be identified that, by inference or (as proposed in this study) by statistical assessment, can be attributed to specific forcing mechanisms.

Let $\boldsymbol{D}_{k}$ represent a set of observation vectors at the each grid-point for $N$ observations in time. $\boldsymbol{D}_{k}$ may be then expressed by using summation notation as:

$\boldsymbol{D}_{k}=\sum_{i=1}^{\mathrm{M}} y_{i k} \boldsymbol{E}_{i} \quad k=1,2, \cdots, N$

where $M$ is the total number of grid-points, $\boldsymbol{E}_{i}$ the eigenvector associated with the $i^{\text {th }}$ eigenvalue and $y_{i k}$ is the time-dependent coefficient (score) of the $i^{\text {th }}$ eigenvector for the $k^{\text {th }}$ observation in time. In our case, the total number $N$ of observations is equal to 365 and $M$ represents the 40 grid-points. Hence, 40 eigenvalues and eigenvectors are produced. Generally, the most important (first one) eigenvector tends to describe the $D$ region with largest fluctuation (generally, where $D$ is greatest).

Spatial maps of these principal components and their interpretation can be enhanced by rotation. A few desirable qualities of rotated solutions for regionalization works include the domain shape independence and that it maximizes the correlation of a few variables with a given principal component PC, at the detriment of explaining total variance across the data set (Karl et al., 1990). Rotation also allows easier interpretation of the PCs. Indeed, both unrotated and orthogonally rotated solutions (Varimax) were computed for $D$, but interpretation of the rotated solutions becomes much more straightforward because it may aid in identifying unique $D$ fields. PCAs advantage of maximizing the variance in a few (a number much smaller than the number of variables, or grid-points in this case) linear combinations of variables dictates that the number of PCs to retain for rotation must be decided prior to rotation. One technique, commonly referred to as "rule N", compares eigenvalues to a distributions of uncorrelated Gaussian variables. For a complete description of the method see Preisendorfer (1988). In general, rule $\mathrm{N}$ seemed overly conservative retaining only a few (three, herein) components. The first unrotated and rotated components approximately explained 78 and $36 \%$ of the total variance, respectively (Table 2 ). The first rotated component was therefore of most interest for describing diabatic processes operating over the Iberian Peninsula for most explained variance reasons. It would appear, then, to be reasonable that the case study would be one bearing the strongest resemblance to the first rotated PC. 
Table 2. Variances $\lambda_{i}$ and cumulative variance ratios (percentages) of the first three PCs

\begin{tabular}{|c|c|c|c|c|c|c|}
\hline & \multicolumn{2}{|l|}{ 1st PC } & \multicolumn{2}{|l|}{ 2nd PC } & \multicolumn{2}{|l|}{ 3rd PC } \\
\hline & Nonrotated & Rotated & Nonrotated & Rotated & Nonrotated & Rotated \\
\hline Variance explained & 31.1 & 14.2 & 3.6 & 13.0 & 2.4 & 9.8 \\
\hline $\begin{array}{l}\text { Cumulative proportion } \\
\text { of total variance }\end{array}$ & 78 & 36 & 87 & 69 & 93 & 93 \\
\hline
\end{tabular}

To elucidate this requirement, Molteni et al. (1983) procedure has been used here. At root, Molteni's approach is intended to obtain the rotated first-PC score corresponding to the $k^{\text {th }}$ day that the projection of the data vector $\boldsymbol{D}_{k}$ onto the first rotated eigenvector is a maximum. To do this, a two stage classification is conducted as follows:

1. Only the scores $y_{i k}^{r}$ of the first rotated PC whose values are higher than an empirical threshold are retained. Put mathematically, the following conditions are to be held for:

$z_{l k}^{r} \geq 1.5$ and $\frac{z_{l k}^{r}}{\max \left(z_{j k}^{r}\right)} \geq 2 j \neq 1$

where $z_{l k}^{r}=y_{l k}^{r} / \lambda_{l}^{1 / 2}$ is the normalized score, being $\lambda_{l}^{1 / 2}$ the standard deviation of the first rotated principal component;

2. In such a discriminated score set, the score most conformed to the first rotated PC was isolated: correlations between the $700 \mathrm{hPa}$ averged $D$-field and each of the $700 \mathrm{hPa}$ individual $D$-fields were computed. It is the individual $700 \mathrm{hPa} D$-field showing maximum correlation with the joint discriminated averaged field that was chosen as a snapshot of the first rotated PC.

\section{The example of 15 March 1990}

The implementation of the methodology allowed the 15 March 1990 atmospheric situation to be objectively

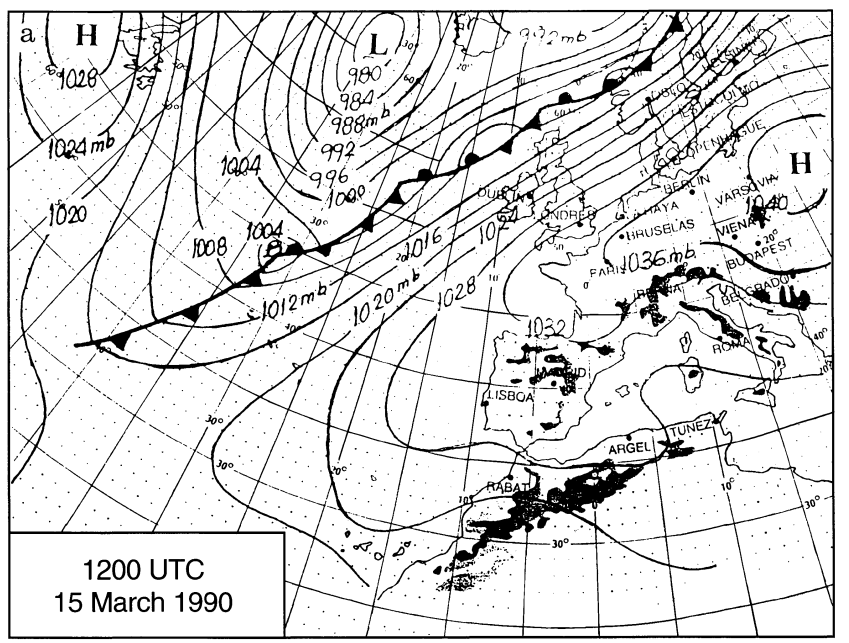

selected to analyze the diabatic forcing obtained by means of the proposed parametrization.

The selected day corresponds to the synoptic pattern characterized by a stationary, high-amplitude ridge, i.e., by a blocking high as viewed in Fig. 2. Despite the fact that such a situation may be considered rather special (since blocking situations usually occur on $10 \%$ of all days over western Europe, Tibaldi et al., 1994), such situations are of interest because of their profound effect on local and regional climates and particularly on southwestern Europe.

On the surface chart (Fig. 2a), an anticyclone centered over Central Europe extended over the west of Europe. A deep cyclone with a $980 \mathrm{mb}$ center was located west of Iceland possessing a secondary low as a pivotal point for a cold front extending southwestward to the Azores Islands. Figure $2 \mathrm{~b}$ shows the $500 \mathrm{hPa}$ geopotential analysis for the same day. The surface low pressure center, situated to the west of Iceland, is also present at $500 \mathrm{hPa}$ while a ridge stretched along a tilted SW-to-NE axis through Iberian. Also of remarkable interest is the existence of a weak but very significant low over Sardinia.

In such a situation, strongly stable conditions prevail over most of the Iberian Peninsula, except for the zone of Balearic Islands and eastern Iberia where stratiform cloudiness was reported (Fig. 3). Unlike the 1200 UTC Palma de Mallorca and Murcia soundings, which show unstable conditions (Fig. 4), the remaining soundings over Iberian Peninsula (not shown) are not representative of such conditions. Both soundings depict a moist layer below $700 \mathrm{hPa}$ separated from a dry layer above by

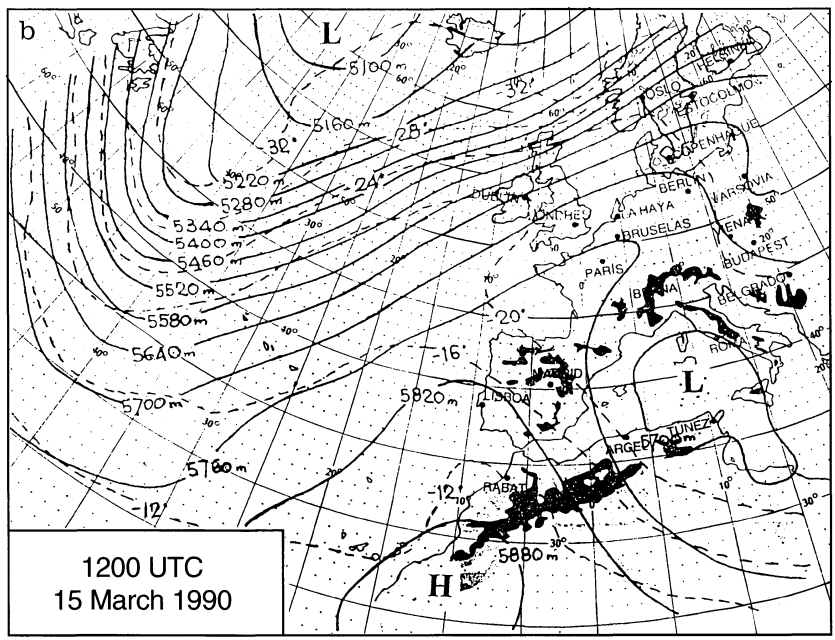

Fig. 2a,b. Synoptic situation at 1200 UTC on 15 March 1990. a Surface analysis (pressure contours every 4 mb); b 500 hPa analysis (height contours, every $60 \mathrm{~m}$, continuous lines; temperature contours, every $4{ }^{\circ} \mathrm{C}$, dashed lines) 


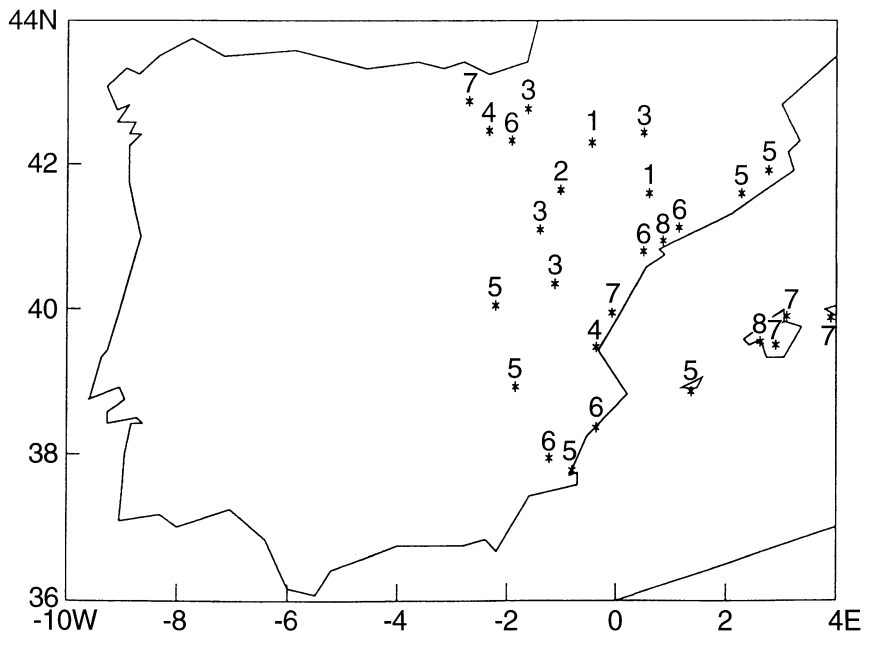

Fig. 3. Spatial pattern of cloudiness for 15 March 1990. Numbers indicate sky covered by cloud in oktas

a shallow stable layer with inversion. Furthermore, it can be observed in the Palma de Mallorca sounding a moderate convective available potential energy (CAPE) of $404 \mathrm{~J} \mathrm{Kg}^{-1}$ that suggests convective instability. A northerly flow around the western flank of the low over Sardinia affects completely eastern Spain. In fact, Fig. 4a highlights this northerly flow. It is apparent that the atmosphere has an approximately equivalent barotropic structure in the troposphere. Lińes (1970) asserts that a northerly flow over western Mediterranean is a condition well suited for rainfall over such a zone. Indeed, the precipitation field (Fig. 5) displays some daily rainfall (lower than $1 \mathrm{~mm}$ ) associated with stratiform cloudiness over the Balearic area and the absence of it over Iberia.

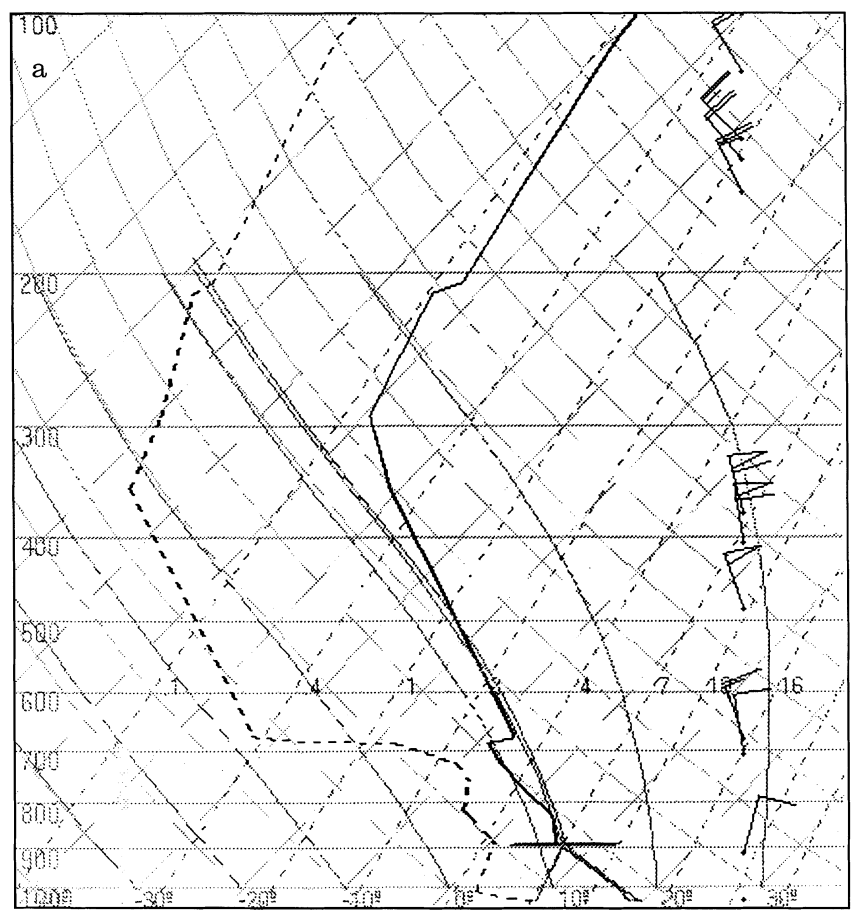

The computed quasigeostrophic forcing, $G$, for vertical motion is displayed in Fig. 6 for $700 \mathrm{hPa}$ at 1200 UTC. Relatively moderate positive values $\left(\approx 8 \times 10^{-17}\right.$ $\mathrm{hPa}^{-1} \mathrm{~s}^{-3}$ ) are located to the northeast of Iberia. This area is directly affected by northerly air flow brought about by Sardinia's low and convergence of $\mathrm{Q}$ vectors results. The remaining area of Iberian Peninsula shows negative, relatively moderate values associated with subsidence at the high pressure area.

On the other hand, a composite $700 \mathrm{hPa}$ diabatic forcing, $D$, is displayed in Fig. 7. It is noticeable that there is a region with positive values (about $12 \times$ $10^{-17} \mathrm{hPa}^{-1} \mathrm{~s}^{-3}$ which means a relatively strong diabatic heating associated with latent heat release over eastern Iberia and Balearic Islands. By comparing Fig. 7 and Fig. 3, it can be observed how the area of positive diabatic heating matches the cloudiness area reported on this date. Such a feature may well be due to the inclusion of the ice-liquid water potential temperature in the diabatic heating parametrization since for adiabatic conditions (Fig. 6) it does not occur. Moreover, the strongest diabatic heating area seems to be quite realistic when compared to the precipitation area in Fig. 5. The remainder region of the data window shows distinctly negative values $\left(\approx-13 \times 10^{-17} \mathrm{hPa}^{-1} \mathrm{~s}^{-3}\right)$ that denote moderate diabatic cooling and favor downdrafts. This is consistent with the mentioned strongly stable conditions stretching over much of Iberia.

\section{Concluding remarks}

As already mentioned this study basically focused on the question of how the diabatic forcing term is to be applied

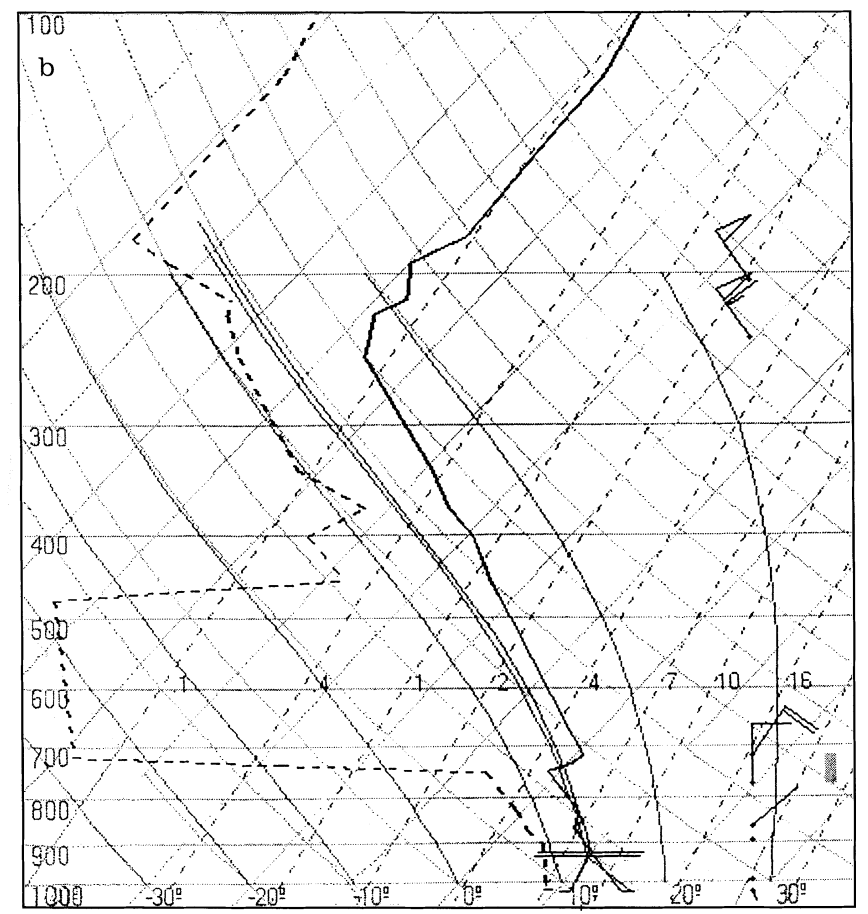

Fig. 4a,b. Skew $T$ - $\log p$ plot for 1200 UTC on 15 March 1990 for a Palma de Mallorca sounding; b Murcia sounding. Solid line is the temperature; dashed line is the dewpoint. Wind barbs are in knots 


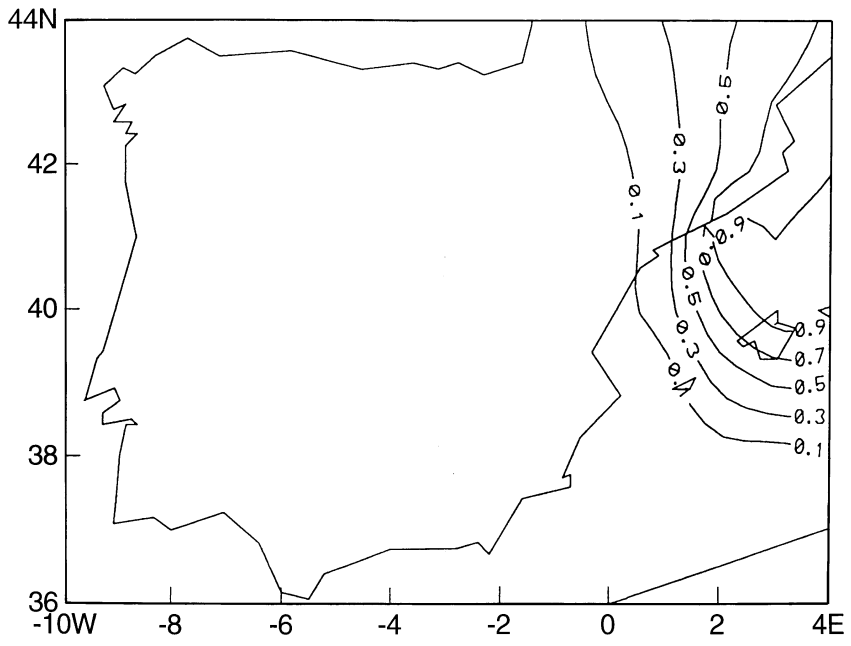

Fig. 5. Isohyets of daily rainfall (mm) for 15 March 1990

accurately in the quasigeostrophic omega equation over Iberia. Certain aspects of this subject have been examined. A formulation of diabatic term, including all phase changes of water, has been considered in this study.

$G$ and $D$ were computed in 40 grid-points over Iberia and Balearic Islands from Spanish Sounding Network for every day 1990 and for a number of mandatory levels. By studying the space-time averages of $D$ and $G$ absolute values at $850,700,500,400$ and $250 \mathrm{hPa}$, it can be observed that the order of magnitude is the same for the two types of forcing and that $D$ is greater than $G$ below the $500 \mathrm{hPa}$ level. We also compared the calculated outputs of the diabatic term against the dynamic forcing term in the omega equation for an objectively chosen case.

The chosen case study was selected by applying a methodology aimed at finding a spatial $D$ pattern for diabatic term whose resemblance is the largest when compared to the first rotated PC for the $D$ field. The situation for 15 March 1990 is identified as that corresponding to the first principal component. The related

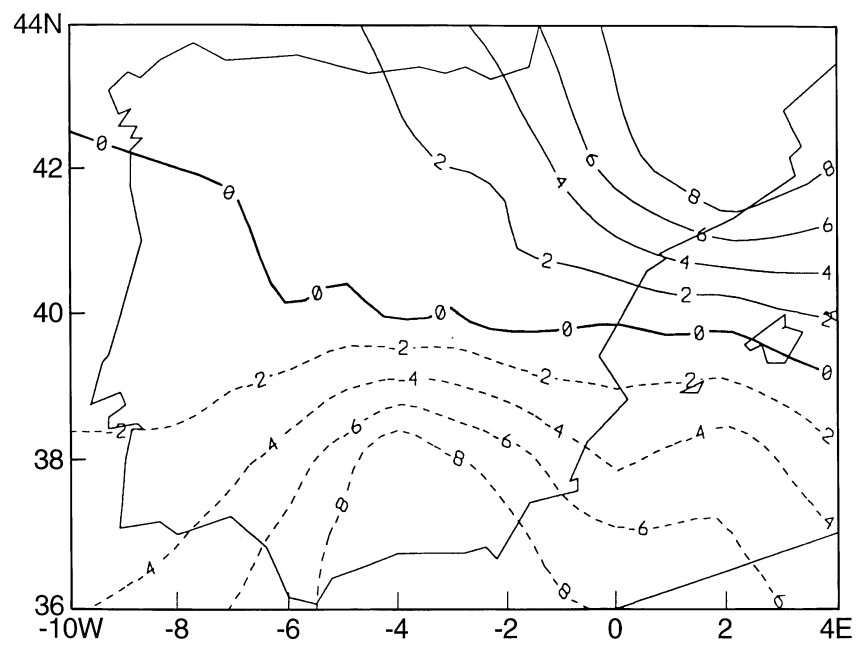

Fig. 6. Divergence of the Q-vectors (times -2) at $700 \mathrm{hPa}$ for 1200 UTC 15 March 1990. Contour interval is $2 \times 10^{-17} \mathrm{hPa}^{-1} \mathrm{~s}^{-3}$

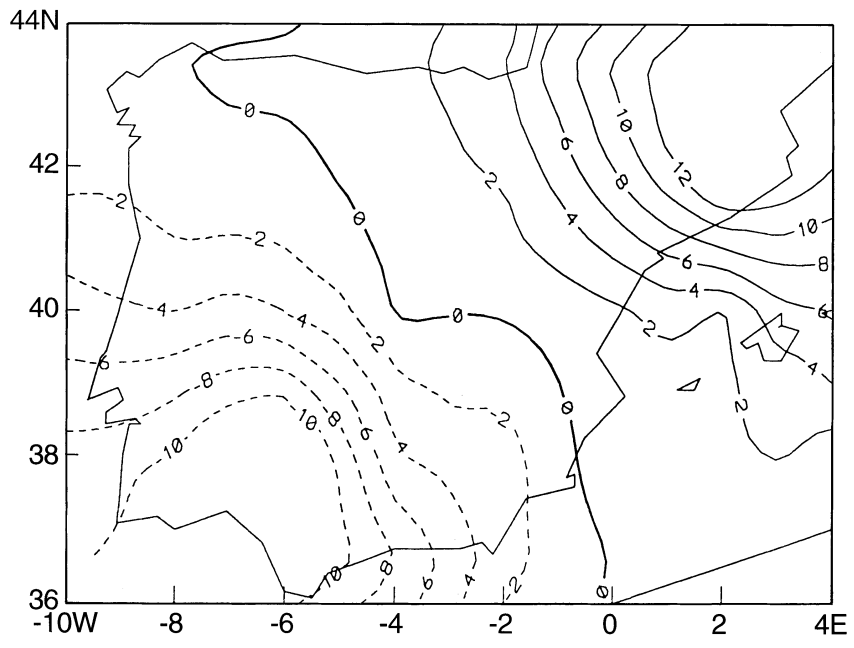

Fig. 7. Diabatic heating at $700 \mathrm{hPa}$ for 1200 UTC 15 March 1990 Contour interval is $2 \times 10^{-17} \mathrm{hPa}^{-1} \mathrm{~s}^{-3}$

synoptic situation is characterized by a stationary, highamplitude ridge which produces strongly stable conditions over much of Iberia. Eastern Iberia and Balearic Islands are, on the contrary, undeniably affected by a low situated over Sardinia which induces a northerly air flow and brings cloudiness and precipitation into such a zone. At $700 \mathrm{hPa}$ level, the $D$ term is significantly greater than the $G$ term and therefore the $D$ term must be considered when applied the quasigeostrophic formulation in Iberia. The $D$ spatial distribution matches the observed rain and cloud pattern to confirm the skill of the diabatic forcing parametrization developed in this study.

One might reasonably suppose that by including only a slightly further level of sophistication in the classical quasigeostrophic omega equation by adding the described parametrization, it might well gain some insight into rain and cloudiness features over Iberia. However, the checking of this assumption would have to depend on more specific research which, with almost complete certainty, would lead to a rather different study.

Acknowledgements. The authors would like to thank the Spanish Meteorological Institute for supplying the data. This research was made possible in part by a FPI Grant from the Ministerio de Educación y Ciencia of Spain. We are also very much indebted to the referees for their fruitful discussions concerning this work.

Topical Editor L. Eymard thanks M. Fontini and another referee for their help in evaluating this paper.

\section{Appendix A}

\section{List of symbols}

$C_{p} \quad$ specific heat of dry air at constant pressure

$D \quad$ diabatic forcing term, i.e., $-\frac{R}{p} \nabla_{\mathrm{p}}^{2}\left(T \frac{\mathrm{d} \ln \theta_{i l}}{d t}\right)$

$E_{i} \quad$ eigenvector associated with the $i^{\text {th }}$ principal component

$f \quad$ Coriolis parameter

$G \quad$ dynamic forcing term, i.e., $\left(-2 \nabla_{p} \cdot \mathrm{Q}\right)$ 
$J \quad$ rate of heating per unit mass

$L_{i v} \quad$ latent heat of sublimation

$L_{l v} \quad$ latent heat of vaporization

$p \quad$ total pressure of air

Q Q vector

$R \quad$ dry air gas constant

$r_{i} \quad$ mixing ratio of ice water

$r_{l} \quad$ mixing ratio of liquid water

$s \quad$ specific entropy

$t$ time

$T$ temperature

$y_{i k} \quad$ score of the $i^{\text {th }}$ principal component in the $k^{\text {th }}$ day

$y_{i k}^{r} \quad$ score of the $i^{\text {th }}$ rotated principal component in the $k^{\text {th }}$ day

$z_{i k}^{r} \quad$ standardized score of the $i^{\text {th }}$ rotated principal component in the $k^{\text {th }}$ day

$\lambda_{i}^{1 / 2}$ standard deviation of the $i^{\text {th }}$ principal component

$\theta \quad$ potential temperature of air

$\theta_{l} \quad$ liquid water potential temperature

$\theta_{m} \quad$ potential temperature of moist air

$\theta_{i l}$ ice-liquid water potential temperature

$\sigma \quad$ static stability parameter

$\omega$ vertical motion

\section{References}

Betts, A. K., Non-precipitating cumulus convection and its parameterization. Q. J. R. Meteorol. Soc. 99, 178-196, 1973.

Bosart, L. F., Weather forecasting, in Handbook of applied meteorology Ed. D. D. Houghton, Wiley New York, 205-280, 1985.

Chang, C. B., D. J. Perkey, and C. W. Kreitzberg, Latent heat induced transformations during cyclogenesis, Mon. Weather Rev., 112, 357-367, 1984.

Chen, S.-J., L.-J. Bai, and S. L. Barnes, Omega diagnosis of a cold vortex with severe convection, Wea. Forecast., 3, 296-304, 1988.

Hauf, T. and H. Höller, Entropy and potential temperature, J. Atmos, Sci. 44, 2887-2901, 1987.

Holton, J. R., An introduction to dynamic meteorology, 1st Ed., Int. Geophys. Ser., 23, Academic Press, 319 pp, 1972.
Hoskins, B. J., and M. A. Pedder, The diagnosis of middle latitude synoptic development, Q. J. R. Meteorol. Soc., 106, 707-719, 1980.

Hoskins, B. J., I. Draghici, and H. C. Davies, A new look at the (-equation. Q. J. R. Meteorol. Soc., 104, 31-38, 1978.

Iribarne, J. V., and W. L. Godson, Atmospheric thermodynamics, Geophys. Astrophys. Monogr. 6, Reidel, 222 pp, 1973.

Kari, T. R., W. Wang, M. E. Schlesinger, R. W. Knight, and D. Portaman, A method of relating general circulation model simulated climate to the observed local climate. Part I: Seasonal statistics, J. Clim. 3, 1053-1079, 1990.

Linés, A., The climate of the Iberian Peninsula, World survey of climatology, vol 5: Climate of Northern and Western Europe, Ed. C.C. Wallén, Elsevier, Dordrecht, 195-239, 1970.

Lips, F. B., and R. S. Hemler, Another look at the thermodynamic equation for deep convection, Mon. Weather Rev., 108, 78-84, 1980.

Molteni, F., P. Bonelli, and P. Bacci, Precipitation over Northern Italy: a description of means of principal component analysis, J. Clim. Appl. Meteorol., 22, 1738-1752, 1983.

Preisendorfer, R. W., Principal component analysis in meteorology and oceanography, Elsevier Science Publishers B. V. 425 pp, 1988.

Richman, M. B., A cautionary note concerning a commonly applied eigenanalysis procedure, Tellus, 40B, 50-58, 1988.

Ripley, B. D., Spatial statistics. John Wiley, New York, 252 pp, 1981.

Smith, P. J., Role of latent heat release in extratropical cyclone development, Proceeding of a workshop on diabatic forcing, Reading, UK, ECMWF, 245-274, 1987.

Smith, P. J, P. M. Dare, and S. J. Lin, The impact of latent heat release on synoptic-scale vertical motions and the development of an extratropical cyclone system, Mon. Weather Rev., 112, 2421-2430, 1984.

Tibaldi, S., E. Tosi, A. Navarra, and L. Pedulli, Northern and Southern hemisphere seasonal variability of blocking frequency and predictability, Mon. Weather Rev., 122, 1971-2003, 1994.

Trenberth, K. E., On the interpretation of the diagnostic quasigeostrophic omega equation, Mon. Weather Rev., 106, 131-137, 1978.

Tripoli, G. J., and W. R. Cotton, The use of ice-liquid water potential temperature as a thermodynamic variable in deep atmospheric models, Mon. Weather Rev., 109, 1094-1102, 1981.

Yip, T. C., and H. R. Cho, Dynamics of quasi-geostrophic systems with cumulus convection, Tellus 34, 63-73, 1982. 